\title{
Intelligent Lighting System Based on Arduino and Yeelink Platform Yiheng $\mathrm{He}^{1, \mathrm{a}}$, \\ ${ }^{1}$ School of North China Electric Power University, Baoding 071003, China. \\ a619132065@qq.com
}

Keywords: Arduino, Yeelink, intelligent

\begin{abstract}
This paper designs a intelligent lighting system based on Arduino hardware platform and Yeelink cloud platform, and realizes remote control of the system through Android terminal devices. The system is low-cost and easy to set up, and have a friendly UI and other good features, which is suitable for promotion and application.
\end{abstract}

\section{Introduction}

With the development of technology, smart home has an increasingly closer relationship with human's daily life. Meanwhile, people have more requirements of simplified and humanized operations of smart home system [1].

Smart home takes advantage of advanced computer technology, network communication technology, premises distribution technology, medical electronics technology, conforms to the ergonomic principle, integrates with individual needs, combines each subsystem related to home life together, such as lighting control, multimedia home appliances control as well as kitchenware control, and implements control and management through network and mobile terminal devices to achieve "people-oriented" and completely new home experience[2]. Among them, lighting control is most closely related to daily life. At present, most lighting controls still use relatively more traditional mechanical switches controls, which are cumbersome and inefficient, so they are not in line with the development trend of intelligent home. While some existing lamps with remote control mainly adopt infrared remote controller to realize remote control. Although this control mode enjoys a lower cost, the remote control device only has remote control effects on paired lamps, so it does not have generality. In the case of a large number of lamps, this mode is difficult to achieve flexible and convenient effects. In addition, at present, companies including Millet have launched intelligent lighting systems, which are too expensive and hard to be promoted, so the development of intelligent lighting is restrained.

Therefore, this paper designs an intelligent lighting system based on Arduino UNO hardware platform. The system uses Yeelink, a nationwide well-known IOT platform, as the back end, and takes iPhone, Android phones and other smart terminal APP as the controller. The scheme enjoys a low cost, good expansibility, convenient use and so on.

\section{System components}

The system mainly takes main control board Arduino UNO as the hardware platform, whose cooperation with the network extension board Arduino Ethernet W5100 and electromagnetic relays forms the hardware system. Meanwhile, the system employs cloud platform Yeelink as a service back end, and uses devices based on intelligent terminal of iOS and Android systems as control devices. In the system, the control terminal and the server, as well as the server and the user terminal are connected via wireless network, thus ensuring the portability of handheld terminal and the continuity of data exchange[1].

Arduino platform. Arduino was created jointly by David Cuartielle and Massimo Banzi, two teachers of Milan Interaction Design Institute in 2005. Arduino is a Simple I/O platform [3] based on the start code, open source, and consisting of two parts---hardware and software. This design uses the Arduino UNO platform, as shown in Figure 1, and its core component is a piece of AVR 
AT-mega128MCU. Compared with other master control platforms of different specifications, the open source and simplicity are its core advantages. The platform being used can also be downloaded for free. The design uses the integrated and modular designs, and integrates the Arduino open source module, relay control circuit and Arduino Ethernet W5100 wireless network module into a controlled end, so as to achieve the portability, integration and easy installation of the controlled end [1].

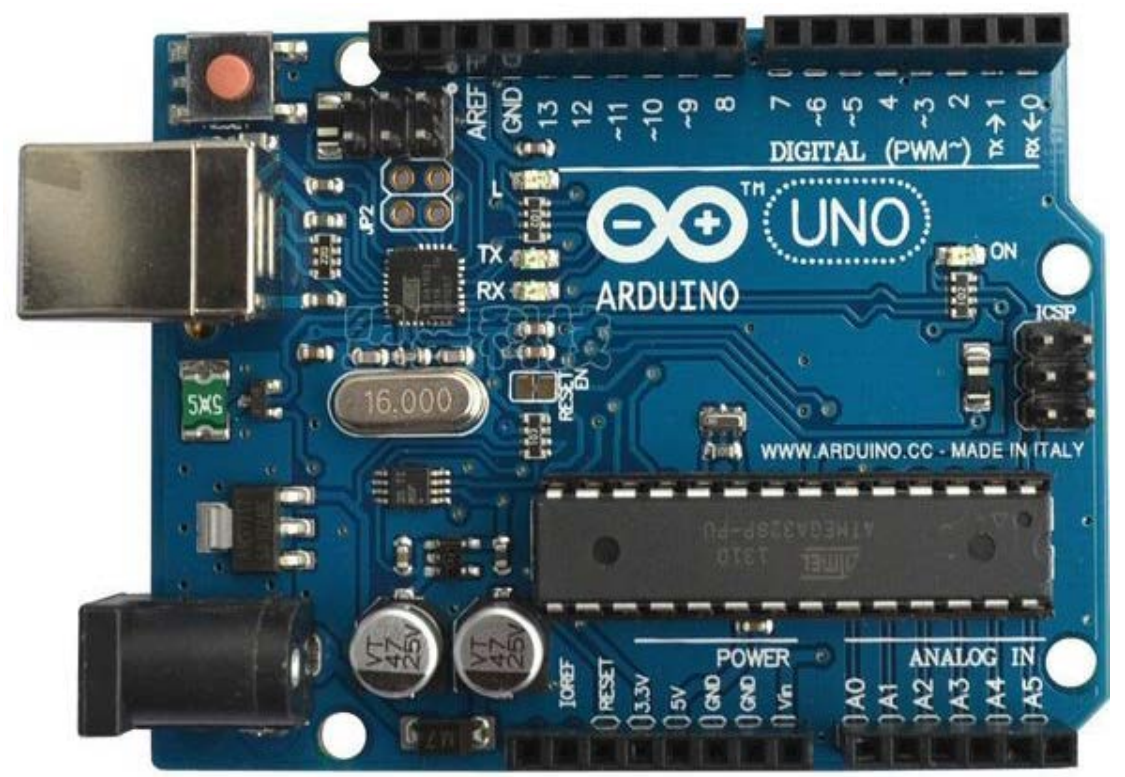

Figure 1 Arduino UNO main control board

In this design, the wireless extension module applies Arduino Ethernet W5100. The module can make the Arduino module be connected with Yeelink platform through the wireless router, so as to achieve the communication between the cloud computing platform and remote terminal.

Yeelink platform. Yeelink is one of the earliest Internet service providers which provides free sensor data access management [4]. In its control interface of Web side, users can complete the control, access and other operations of switch, sensor, and other equipment, and at the same time, the platform can present the related data to users timely. The platform allows users to complete the storage of mass sensor data, and trigger a series of actions according to users' settings, such as sending e-mails or micro-blog information, and also realizing the control function of household electrical appliances [1].

User operation terminal. In this paper, the APP based on smart phone system is used for the remote control of lamps, and in the following text, the related experiments are done with smart phone of Android system as an example. According to relevant data, it is showed that the popularizing rate of smart phones in China has reached 66\% [2]. As a mobile terminal with high usage rate, the smart phone has the characteristics of operability, strong expansibility, easy networking, etc., and conforms to the intelligent home's requirements for remote control device. Therefore, the research on the development of intelligent lamps with Android smart phones as operation terminals has a very broad market prospect.

\section{System design}

Hardware circuit. The hardware module of this design takes Arduino UNO as the main platform, and the platform accesses to the wireless local area network through W5100. The hardware circuit is shown in Figure 2, in which the relay belongs to the "normally open" relay. The Arduino main control board is connected to current-limiting resistance of $1.2 \mathrm{k} \Omega$ externally and is connected to bases of light emitting diode and VT9013 triode through a pin. The electromagnetic relay is connected to $+5 \mathrm{~V}$, a voltage source and the VT9013 collector, while the emitter of VT9013 is grounded. This design completes the control of lamps' turn-on and turn-off according to the principle as described below: When the stitch of Arduino is at the high potential, the work of VT9013 is in saturation condition, 
$V_{C E} \approx 0 \mathrm{~V}$ equivalent to the switch on, then the electromagnetic relay is switch on, and lamps are in a working state with electricity; when the stitch of Arduino is at the low potential, the work of VT9013 is in the cut-off state, $V_{C E} \approx 5 \mathrm{~V}$ equivalent to the switch off, the electromagnetic relay is switch off, the $220 \mathrm{~V}$ circuit to which lamps are connected is in the open-circuit state, and lamps are turned off.

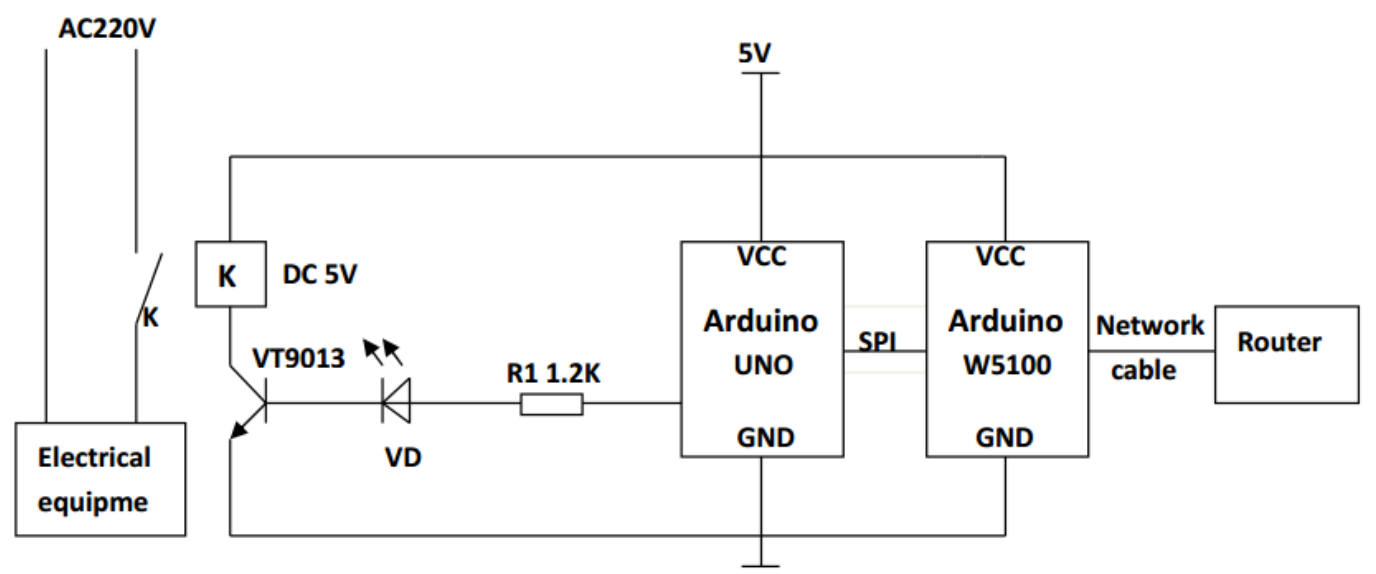

Figure 2 Circuit diagram of hardware system

Program design. The programming language of Arduino is similar to $C$ language. As the open source on Arduino provides common library files in advance in integrated software development environment (IDE), while using its basic functions people only needs to download all needed libraries on sharing platform Github and then call [5]. The library contains plenty of common functions, such as I/O control, etc. Besides, it is very accessible, which allows developer to focus on program design rather than distracting themselves on learning development language.

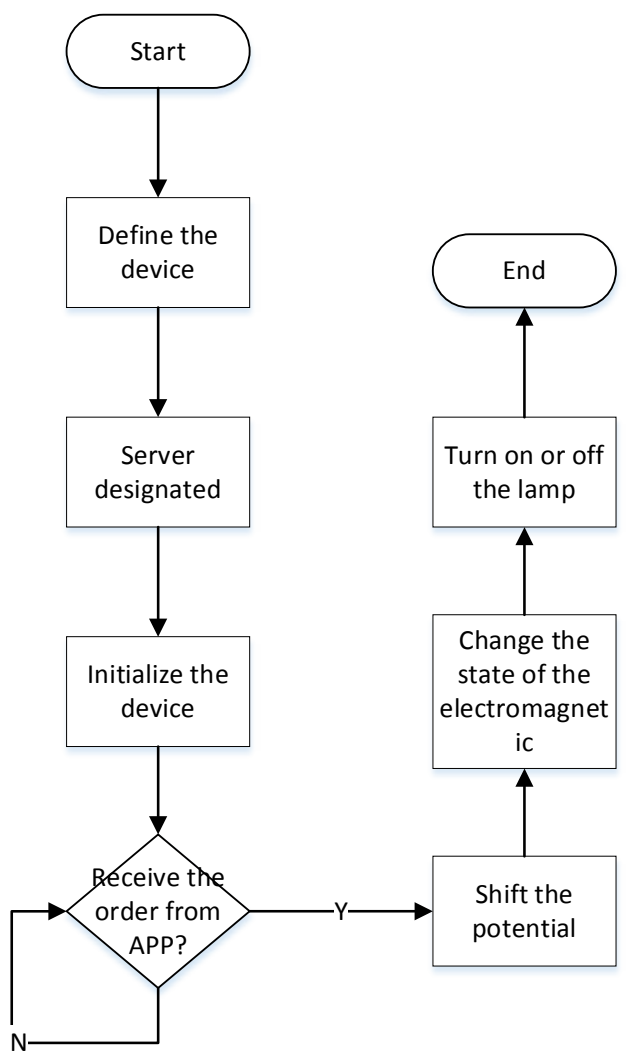

Figure 3 Software flow diagram

Figure 3 shows the main flow of the program. 
We use the following loop subfunction as an example to introduce the control program. The loop checks whether the Arduino system receives the control order from the server. If the order of the switch state changes, it will adjust stitch potential; if not, then repeat the judgment statement.

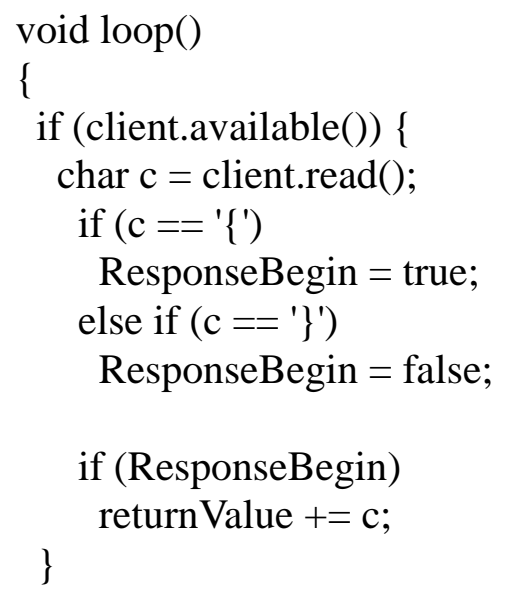

The local hardware would be carried out matching operation with Yeelink server side after debugging and testing the local program. As matching operation is somewhat simple, this paper does not make detailed description herein.

Operation interface. After debugging, testing and matching, users can realize the remote control of switch through the application in intelligent terminal devices. As shown in Figure 4 of the program user interface (UI) of Android system, we can see that the UI is easy and accessible to users.

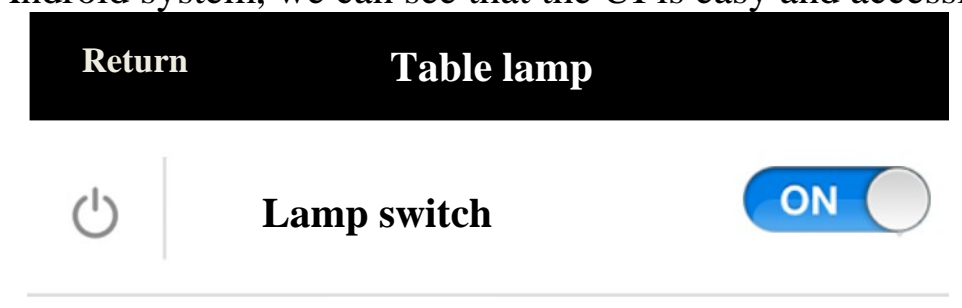

Figure 4 Android client UI

\section{Conclusion}

The paper successfully sets up a minitype intelligent lamplight control system based on open source Anduino platform and Android intelligent terminal, achieving satisfied effect in practical application and having a certain reference value for electronics enthusiasts to build personalized platform. Due to the utility of open source environment, the system can be built easily and rapidly and its effect is no less than a commercial system [1]. In addition, this system has strong expandability, which can realize more advanced functions, and its control methods could be extended into other intelligent systems, home appliances and remote control of devices.

\section{References}

[1] Wang Zhen, Yang Xiaolan. Intelligent home system design based on Android and Arduino [J]. Software Guide, 2014, ?(12):94-96.

[2] Information on http://baike.baidu.com

[3] Cai Ruiyan. Principle and application of Arduino [J]. Electronic Design Engineering, 2012, ?(16):155-157

[4] Information on http://www.yeelink.net

[5] Yuan Benhua, Dong Zheng. Design of the Temperature Measurement System Based on Arduino Board [J]. Journal of Anhui Agri. Sci., 2012, 08:5049-5050 